\title{
B-type supergiants in M 31
}

\author{
Carrie Trundle ${ }^{1,2}$, Philip L. Dufton ${ }^{1}$, Daniel J. Lennon ${ }^{2}$, \\ Stephen J. Smartt ${ }^{3}$, and Miguel A. Urbaneja ${ }^{4}$ \\ 1 Department of Pure and Applied Physics, The Queen's University, \\ Belfast BT71NN, Northern Ireland \\ ${ }^{2}$ The Isaac Newton Group of Telescopes, Apartado de Correos 321, \\ E-38700 Santa Cruz de La Palma, Canary Islands, España \\ ${ }^{3}$ Institute of Astronomy, University of Cambridge, \\ Madingley Road, Cambridge CB30HA, UK \\ ${ }^{4}$ Instituto de Astrofísica de Canarias, \\ C/ Vía Láctea s/n, E-38200 La Laguna, Tenerife, España
}

\begin{abstract}
We present the spatial distribution of chemical species in M 31, as derived from intermediate resolution spectra of seven B-type supergiants, lying within four $\mathrm{OB}$ associations, covering a galactocentric distance of $5-12 \mathrm{kpc}$. We do not detect any systematic oxygen gradient across this galactocentric range. We find that the inner regions of M 31 are not, as previously thought, very 'metalrich'. Our abundances of $\mathrm{C}, \mathrm{N}, \mathrm{O}, \mathrm{Mg}, \mathrm{Si}, \mathrm{Al}, \mathrm{S}$ and $\mathrm{Fe}$ in the M 31 supergiants are very similar to those of massive stars in the solar neighbourhood.
\end{abstract}

\section{Discussion}

Most previous studies on abundance gradients in external galaxies are based on $\mathrm{H}$ II regions and supernova remnants (SNRs). However, the reliability of the abundances measured from low excitation nebulae, such as those in the inner regions of $\mathrm{M} 31$, is as yet unclear. In these nebulae, empirical calibrations are implemented to estimate abundances. As evident from Figure 1, the derived abundances from $\mathrm{H}$ II regions, and hence the radial gradients, have a strong dependence on the empirical calibration adopted (see Pagel et al. 1980; Mc Gaugh 1991; Zaritsky et al. 1994; Pilyugin 2001). In the case of M 31, a difference in oxygen abundance of $\sim 0.56 \mathrm{dex}$ and radial oxygen gradient of $-0.013 \mathrm{dex} \mathrm{kpc}^{-1}$ is obtained by using four different empirical calibrations on a group of $11 \mathrm{H}$ II regions from Blair et al. (1982). Due to this ambiguity, an independent method of investigating the spatial distribution of elements in distant galaxies is necessary. Fortunately, B-type supergiants provide us with such a diagnostic tool.

From intermediate resolution spectra, we derived the chemical composition of seven B-type supergiants in M31. The radial abundance gradients of oxygen, silicon and magnesium indicate towards a negligible abundance gradient and solar metallicity in the inner regions of the galaxy (see Figure 1). There is an offset between the oxygen abundances obtained from the $\mathrm{H}$ II regions and that of the B-type supergiants $(\sim 0.15-0.4 \mathrm{dex})$, at the same galactocentric distance, which depends on the empirical calibration implemented. 


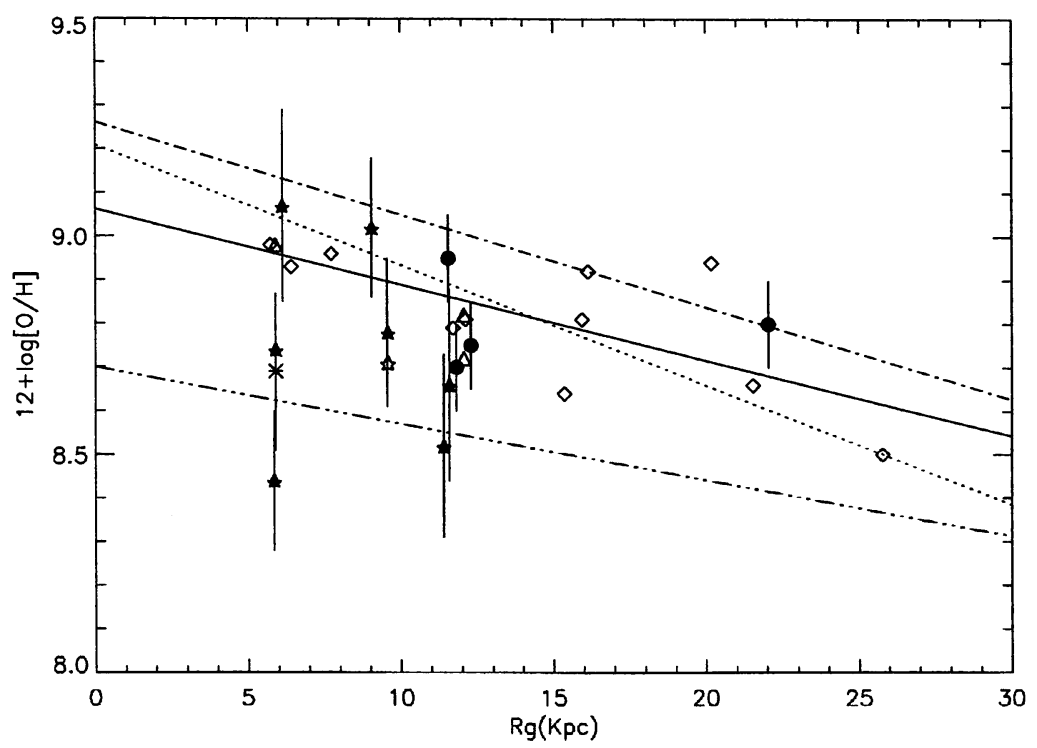

Figure 1. Oxygen abundances for H II regions and supergiants in M31 as function of galactocentric distance. Solid stars $(\star)$ : abundance results of the seven B-type supergiants in this study. Unfilled star: non-LTE oxygen abundance for OB78-277. Asterisk (*): photospheric non-LTE abundance of OB1064 (Smartt et al. (2001). Open diamonds $(\diamond)$ and open triangles $(\triangle)$ : abundances of the $\mathrm{H}$ II regions from Blair et al. (1982) and Galarza et al. (1999), respectively, calculated using McGaugh (1991) calibrations. Solid circles (•): abundances of four A-F-type supergiants (Venn et al. 2000). Dotted ( ...), solid $(-)$, dash-dot (---) and dash-dot-dot-dot (-. .-) lines: least squares fit through the H II regions of Blair, using Mc Gaugh (1991), Pagel et al. (1980), Zaritsky et al. (1994) and Pilyugin (2001) calibrations, respectively. The error bars in the stellar results represent the standard error in the mean.

Details are given in Trundle et al. 2002.

\section{References}

Blair, W.P., Kirshner, R.P., Chevalier, R.A. 1982, ApJ 254, 50

Galarza, V.C., Walterbos, R.A.M., Braun, R. 1999, AJ 118, 2775

McGaugh, S.S. 1991, ApJ 380, 140

Pagel, B.E.J., Edmunds, M.G., Smith, G. 1980, MNRAS 193, 219

Pilyugin, L.S. 2001, A\&A 369, 594

Smartt, S.J., Crowther, P.A., Dufton, P.L., et al. 2001, MNRAS 325, 257

Trundle, C., Dufton, P.L., Lennon, D.J., Smartt, S.J., Urbaneja, M.A. 2002, A\&A 395, 519

Venn, K.A., McCarthy, J.K., Lennon, D.J., et al. 2000, AJ 541, 610

Zaritsky, D., Kennicutt, R.C., Huchra, J.P. 1994, 420, 872 